YITP-SB-01-12, CITUSC/01-013

\title{
Partition functions and Jacobi fields in the Morse theory
}

\author{
Soon-Tae Hong* \\ C.N. Yang Institute for Theoretical Physics, \\ State University of New York at Stony Brook, Stony Brook, NY 11794, USA \\ and \\ CIT-USC Center for Theoretical Physics, \\ University of Southern California, Los Angeles, CA 90089-2535, USA
}

November 13, 2018

\begin{abstract}
We study the semiclassical partition function in the frame work of the Morse theory, to clarify the phase factor of the partition function and to relate it to the eta invariant of Atiyah. Converting physical system with potential into a curved manifold, we exploit the Jacobi fields and their corresponding eigenvalue equations to be associated with geodesics on the curved manifold and the Hamilton-Jacobi theory.
\end{abstract}

PACS: 02.40.-k, 03.65.Sq, 04.90.+e

Keywords: Morse theory, Jacobi field, partition function, eta invariant

* On leave from Department of Physics, Sogang University, Seoul, Korea E-mail address: sthong@ccs.sogang.ac.kr 
Nowadays there have been considerable discussions concerning the topological invariants such as the Euler characteristics, the Hopf invariant and so on in mathematical physics. Moreover, the eta invariant of Atiyah has been for instance studied by Witten in quantum field theory associated with the Jones polynomial and knot theory [回] and even in hadron physics such as the chiral bag model [2]. Recently, the Yamabe invariant [3, 4] are also investigated in general relativity associated with the topology of boundary surface of black holes with nontrivial higher genus.

On the other hand, since Feynman proposed the path integral formalism in 1948 [5], there have been tremendous developments in quantum field theory. Especially, the partition functions in the path integral scheme have become crucial in investigating many aspects of recent theoretical physics. In fact, Morette studied the partition functions semiclasically long ago, but her expression for its phase factor possesses somehow subtleties [6].

In this paper we reformulate the semiclassical partition function in the frame work of the Morse theory of differential geometry, to clarify the phase factor of the partition function and to relate it to the eta invariant of Atiyah [7, [1]. To do this, we will convert the physical system with potential into a curved manifold, on which we will use the Jacobi fields and their corresponding eigenvalue equations associated with the geodesics on the curved manifold, and the Hamilton-Jacobi theory.

Now we consider a particle in a conserved physical system with constant energy $E$, which consists of the kinetic and potential energies given as $T=$ $m \delta_{i j} v^{i} v^{j} / 2$ with $v^{i}=d x^{i} / d \tau$ and $V=V\left(x^{i}\right)$, respectively. Converting the potential energy into curvature of the target manifold, one can obtain action of the particle of the form $\square$

$$
S=\int_{\tau_{1}}^{\tau_{2}} \mathrm{~d} \tau\left(g_{a b} v^{a} v^{b}\right)^{1 / 2},
$$

to yield a curved three-dimensional manifold $M$ with metric

$$
g_{a b}=\frac{m(E-2 V)^{2}}{2(E-V)} \delta_{a b},
$$

\footnotetext{
${ }^{1}$ On the curved manifold $M$, we will use the abstract index notation $a, b$ which can be converted into the component notation whenever we wish. Here one notes that, without loss of generality, $V\left(x^{i}\right)$ can be chosen to vanish at starting point at $\tau_{1}$. See Ref. [8] for the notations and conventions used here as in Eq. (10).
} 
which does not have any singularities since the denominator of Eq. (2) is positive definite. With the metric $g_{a b}$ in mind, one can define a unique covariant derivative $\nabla_{a}$ satisfying $\nabla_{a} g_{b c}=0$.

Now we consider a smooth one-parameter family of curves $C_{\alpha}(\tau)$, parameterized by a proper time $\tau\left(\tau_{1} \leq \tau \leq \tau_{2}\right)$ such that for all $\alpha$ and $p, q \in M$, $C_{\alpha}\left(\tau_{1}\right)=p, C_{\alpha}\left(\tau_{2}\right)=q$, and $C_{0}$ is a geodesic or a classical path, along which a tangent vector field $v^{a}$ satisfies the geodesic equation

$$
v^{a} \nabla_{a} v^{b}=\frac{\mathrm{d}^{2} x^{i}}{\mathrm{~d} \tau^{2}}+\Gamma_{j k}^{i} \frac{\mathrm{d} x^{j}}{\mathrm{~d} \tau} \frac{\mathrm{d} x^{k}}{\mathrm{~d} \tau}=0
$$

where $\nabla_{a} v^{b}=\partial_{a} v^{b}+\Gamma_{a c}^{b} v^{c}$. Let $\Sigma$ be a two-dimensional submanifold spanned by curves $C_{\alpha}(\tau)$ and we choose $(\tau, \alpha)$ as coordinates of $\Sigma$. The vector fields $v^{a}=(\partial / \partial \tau)^{a}$ and $w^{a}=(\partial / \partial \alpha)^{a}$ are then the tangent to the family of curves and the deviation vector representing the displacement to an infinitesimally nearby curve, respectively. Here one notes that $w^{a}$ can be always chosen orthogonal to $v^{a}$ and vanishes at end-points to yield the boundary conditions,

$$
w^{a}\left(\tau_{1}\right)=w^{a}\left(\tau_{2}\right)=0 .
$$

Since $v^{a}$ and $w^{a}$ are coordinate vector fields, they commute to each other,

$$
£_{v} w^{a}=v^{b} \nabla_{b} w^{a}-w^{b} \nabla_{b} v^{a}=0 .
$$

In the stationary phase approximation where $\left|w^{a}\right|$ is infinitesimally small, one can expand the action (11) around the geodesic $C_{0}$

$$
S=S_{c l}+S^{(1)}[w(\tau)]+\frac{1}{2} S^{(2)}[w(\tau)]+\cdots,
$$

where $S_{c l}=\left.S\right|_{\alpha=0}$ is a classical action, and ${ }^{2}$

$$
\begin{aligned}
S^{(1)}[w(\tau)] & =\int_{\tau_{1}}^{\tau_{2}} \mathrm{~d} \tau w^{a} \nabla_{a}\left(v^{b} v_{b}\right)^{1 / 2} \\
& =\int_{\tau_{1}}^{\tau_{2}} \mathrm{~d} \tau v_{b} w^{a} \nabla_{a} v^{b}
\end{aligned}
$$

\footnotetext{
${ }^{2}$ For simplicity, we will parameterize the curve so that the Lagrangian $L$ is given as $L=\left(g_{a b} v^{a} v^{b}\right)^{1 / 2}=1$ along the geodesic without loss of generality, since the action (11) is parameterization independent. Here we need to put the condition $\alpha=0$ at the end of the calculations of $S^{(1)}[w(\tau)]$ and $S^{(2)}[w(\tau)]$.
} 


$$
\begin{aligned}
& =-\int_{\tau_{1}}^{\tau_{2}} \mathrm{~d} \tau w^{b} v^{a} \nabla_{a} v_{b} \\
S^{(2)}[w(\tau)] & =-\int_{\tau_{1}}^{\tau_{2}} \mathrm{~d} \tau w^{c} \nabla_{c}\left(w_{b} v^{d} \nabla_{d} v^{b}\right) \\
& =-\int_{\tau_{1}}^{\tau_{2}} \mathrm{~d} \tau w^{c} w_{b}\left(\nabla_{c} v^{d} \nabla_{d} v^{b}+v^{d} \nabla_{c} \nabla_{d} v^{b}\right) \\
& =-\int_{\tau_{1}}^{\tau_{2}} \mathrm{~d} \tau g_{a b} w^{a}\left(v^{c} \nabla_{c}\left(v^{d} \nabla_{d} w^{b}\right)+R_{c d e} v^{c} v^{e} w^{d}\right) \\
& =\int_{\tau_{1}}^{\tau_{2}} \mathrm{~d} \tau g_{i j} w^{i} \Lambda^{j}{ }_{k} w^{k}
\end{aligned}
$$

where we have the Sturm-Liouville operator given as

$$
\Lambda_{j}^{i}=-\delta^{i}{ }_{j} \frac{\mathrm{d}^{2}}{\mathrm{~d} \tau^{2}}-R_{k j l}{ }^{i} v^{k} v^{l}
$$

and we have used Eqs. (3) - (5) and the convention for the Riemann curvature tensor for any vector field $v^{a}$ [8]

$$
\left(\nabla_{a} \nabla_{b}-\nabla_{b} \nabla_{a}\right) v^{c}=-R_{a b d}{ }^{c} v^{d}
$$

Now we consider a partition function [9]

$$
Z\left(q, \tau_{2} ; p, \tau_{1}\right)=\int D\left[x^{i}(\tau)\right] e^{i S\left[x^{i}(\tau)\right]},
$$

which, in the stationary phase approximation, contains a widely oscillatory integral [1, 10, 11 and is thus given by contributions from the points of stationary phase. Here one notes that the stationary points precisely construct the geodesic, along which the total energy is constant. Since the above $S^{(1)}[w(\tau)]$ in Eq. (7) vanishes due to the geodesic equation (3), by inserting Eq. (6) into Eq. (11), one can obtain the partition function of the form,

$$
\begin{aligned}
Z\left(q, \tau_{2} ; p, \tau_{1}\right) & =e^{i S_{c l}} Z^{(2)}\left(q, \tau_{2} ; p, \tau_{1}\right) \\
Z^{(2)}\left(q, \tau_{2} ; p, \tau_{1}\right) & =\int D[w(\tau)] e^{i S^{(2)}[w(\tau)] / 2} .
\end{aligned}
$$

Now we define a scalar product

$$
\left(w, w^{\prime}\right)=\int_{\tau_{1}}^{\tau_{2}} \mathrm{~d} \tau g_{i j} w^{i}(\tau) w^{\prime j}(\tau)
$$


to transform the path space of the integral (13) into a Hilbert space which is an external product of two spaces: three-dimensional space of the physical system and an infinite-dimensional Hilbert space of the continuous scalar functions on $\left[\tau_{1}, \tau_{2}\right]$ vanishing at $\tau_{1}$ and $\tau_{2}$. By choosing an orthonormal basis $\left\{u_{\alpha}^{i}(\tau)\right\}$ in this Hilbert space, one can have the deviation vector $w^{i}(\tau)$ in terms of superposition of $\left\{u_{\alpha}^{i}(\tau)\right\}$

$$
w^{i}(\tau)=\sum_{\alpha=1}^{\infty} a^{\alpha} u_{\alpha}^{i}(\tau)
$$

so that, together with Eq. (14), one can rewrite the second order action (8) as follows

$$
S^{(2)}[w(\tau)]=\sum_{\alpha, \beta} c_{\alpha \beta} a^{\alpha} a^{\beta}
$$

where

$$
c_{\alpha \beta}=\int_{\tau_{1}}^{\tau_{2}} \mathrm{~d} \tau g_{i j} u_{\alpha}^{i} \Lambda_{k}^{j} u_{\beta}^{k}=\left(u_{\alpha}, \Lambda u_{\beta}\right) .
$$

Now in order to diagonalize the matrix $c_{\alpha \beta}$ we find an orthonormal basis of eigenfunctions $\left\{u_{\alpha}^{i}(\tau)\right\}$ of the operator $\Lambda$ with eigenvalues $\lambda_{\alpha}$ via the following eigenvalue equations, which is associated with the Morse theory [12, 8, 13] and is also algebraically treated to compute the semiclassical partition function for the secondary Lagrangian [14],

$$
-\Lambda_{j}^{i} u_{\alpha}^{j}+\lambda_{\alpha} u_{\alpha}^{i}=\frac{\mathrm{d}^{2} u_{\alpha}^{i}}{\mathrm{~d} \tau^{2}}+R_{k j l}{ }^{i} v^{k} v^{l} u_{\alpha}^{j}+\lambda_{\alpha} u_{\alpha}^{i}=0
$$

with

$$
u_{\alpha}^{i}\left(\tau_{1}\right)=u_{\alpha}^{i}\left(\tau_{2}\right)=0, \quad(i=1,2,3) .
$$

With the above eigenvalues and eigenfunctions, one can obtain

$$
c_{\alpha \beta}=\lambda_{\alpha}\left(u_{\alpha}, u_{\beta}\right)=\lambda_{\alpha} \delta_{\alpha \beta}
$$

to yield

$$
S^{(2)}[w(\tau)]=\sum_{\alpha} \lambda_{\alpha}\left(a^{\alpha}\right)^{2}
$$

from which one can rewrite the second order partition function (13) as follows

$$
Z^{(2)}\left(q, \tau_{2} ; p, \tau_{1}\right)=J \prod_{\alpha=1}^{\infty} \int_{-\infty}^{\infty} \mathrm{d} a^{\alpha} e^{i \lambda_{\alpha}\left(a^{\alpha}\right)^{2} / 2} .
$$


Here $J$ is the Jacobian defined as

$$
D[w(\tau)]=J \prod_{\alpha=1}^{\infty} \mathrm{d} a^{\alpha}
$$

and is independent of $w(\tau)$ due to the linearity of the transformation (15), so that $J$ can be brought out of the integral symbol.

By taking the vanishing $\epsilon$ limit of the absolutely convergent integral, one can obtain

$$
\lim _{\epsilon \rightarrow 0} \int_{-\infty}^{\infty} \mathrm{d} a^{\alpha} e^{i \lambda_{\alpha}\left(a^{\alpha}\right)^{2} / 2} e^{-\epsilon\left(a^{\alpha}\right)^{2}}=e^{i \pi \operatorname{sign} \lambda_{\alpha} / 4}\left|\frac{2 \pi}{\lambda_{\alpha}}\right|^{1 / 2}
$$

to arrive at

$$
Z^{(2)}\left(q, \tau_{2} ; p, \tau_{1}\right)=J e^{i \pi \sum_{\alpha} \operatorname{sign} \lambda_{\alpha} / 4} \prod_{\alpha}^{\infty}\left|\frac{2 \pi}{\lambda_{\alpha}}\right|^{1 / 2} .
$$

Here note that the phase factor is proportional to $\sum_{\alpha} \operatorname{sign} \lambda_{\alpha}$, which is associated with the eta invariant of Atiyah [7, 1]

$$
\eta=\frac{1}{2} \lim _{s \rightarrow 0} \operatorname{sign} \lambda_{\alpha}\left|\lambda_{\alpha}\right|^{-s}
$$

Now we relate our geodesic to geodesic of a free particle with the same constant energy $E$ to obtain a closed form of the absolute value in Eq. (25). Recalling that the Jacobian $J$ remains unchanged for the unitary transformation $\left\{u_{\alpha}(\tau)\right\} \rightarrow\left\{\hat{u}_{\alpha}(\tau)\right\}$ [14], for the free particle with the metric $\hat{g}_{i j}=\frac{1}{2} m E \delta_{i j}$ which can be obtained from Eq. (2), one can have the partition function

$$
\hat{Z}^{(2)}\left(q, \tau_{2} ; p, \tau_{1}\right)=J e^{i \pi \sum_{\alpha} \operatorname{sign} \hat{\lambda}_{\alpha} / 4} \prod_{\alpha}^{\infty}\left|\frac{2 \pi}{\hat{\lambda}_{\alpha}}\right|^{1 / 2},
$$

whose corresponding eigenvalue equations can be described as

$$
-\hat{\Lambda}^{i}{ }_{j} \hat{u}_{\alpha}^{j}+\hat{\lambda}_{\alpha} \hat{u}_{\alpha}^{i}=\frac{\mathrm{d}^{2} \hat{u}_{\alpha}^{i}}{\mathrm{~d} \tau^{2}}+\hat{\lambda}_{\alpha} \hat{u}_{\alpha}^{i}=0,
$$

with

$$
\hat{u}_{\alpha}^{i}\left(\tau_{1}\right)=\hat{u}_{\alpha}^{i}\left(\tau_{2}\right)=0, \quad(i=1,2,3)
$$


Combination of Eqs. (25) and (27) yields

$$
Z^{(2)}\left(q, \tau_{2} ; p, \tau_{1}\right)=e^{i \pi \sum_{\alpha}\left(\operatorname{sign} \lambda_{\alpha}-\operatorname{sign} \hat{\lambda}_{\alpha}\right) / 4}\left|\frac{\prod_{\alpha} \hat{\lambda}_{\alpha}}{\prod_{\alpha} \lambda_{\alpha}}\right|^{1 / 2} \hat{Z}^{(2)}\left(q, \tau_{2} ; p, \tau_{1}\right) \text {. }
$$

Now we consider a Jacobi equation to express the absolute value of the ratio in Eq. (30) in terms of the initial data at the starting point $p=x^{i}\left(\tau_{1}\right)$, by introducing a smooth one-parameter family of geodesics $\gamma_{\alpha}(\tau)$ on the manifold $M$. Here one can vary the parameter $\alpha \in R$ by infinitesimally changing the direction of the initial velocity $v^{i}\left(\tau_{1}\right)=\mathrm{d} x^{i} / \mathrm{d} \tau\left(\tau_{1}\right)$ at $p$, and also one can choose $\alpha$ and $\tau$ as coordinates of a submanifold $\Sigma_{\gamma}$ spanned by the geodesics $\gamma_{\alpha}(\tau)$ on $M$. Along the geodesic $\gamma_{0}$, one can have tangent vector fields $v^{a}$ and deviation vector field $w^{a}$ which points to an infinitesimally nearby geodesic and vanishes at $p$, namely, $w^{a}\left(\tau_{1}\right)=0$, so that one can have the relative acceleration of the displacement to an infinitesimally nearby geodesic, [?

$$
a^{a}=v^{c} \nabla_{c}\left(v^{b} \nabla_{b} w^{a}\right)=-R_{b c d}^{a} v^{b} v^{d} w^{c},
$$

to yield the geodesic deviation equation

$$
v^{c} \nabla_{c}\left(v^{b} \nabla_{b} w^{a}\right)+R_{b c d}{ }^{a} v^{b} v^{d} w^{c}=\frac{\mathrm{d}^{2} w_{\alpha}^{i}}{\mathrm{~d} \tau^{2}}+R_{k l m}^{i} v^{k} v^{m} w^{l}=-\Lambda^{i}{ }_{j} w^{j}=0,
$$

which is also known as a Jacobi equation and its solution $w^{a}$ is named a Jacobi field on the geodesic $\gamma_{0}$ whose tangent is $v^{a}$. Since the above Jacobi equation (32) is a linear differential equation, the Jacobi field $w^{i}(\tau)$ depends linearly on the inertial data $w^{i}\left(\tau_{1}\right)=p$ and $\mathrm{d} w^{i} / \mathrm{d} \tau\left(\tau_{1}\right)$ at the starting point $p$ to yield

$$
w^{i}(\tau)=T_{j}^{i}(\tau) \frac{\mathrm{d} w^{j}}{\mathrm{~d} \tau}\left(\tau_{1}\right)
$$

where

$$
T_{j}^{i}\left(\tau_{1}\right)=0, \quad \frac{\mathrm{d} T_{j}^{i}}{\mathrm{~d} \tau}\left(\tau_{1}\right)=\delta_{j}^{i}
$$

${ }^{3}$ Differently from Eqs. (7) and (8), one can exploit the fact that all curves involved in $a^{a}$ are geodesics, so that one can use the identity $w^{c} \nabla_{c}\left(v^{b} \nabla_{b} v^{a}\right)=0$. 
and $T_{j}^{i}(\tau)$ can be defined ast

$$
T^{i}{ }_{j}(\tau)=\frac{\mathrm{d} x^{i}(\tau)}{\mathrm{d} v^{j}\left(\tau_{1}\right)}
$$

Substituting Eq. (33) into Eq. (32), one can rewrite the Jacobi equation in terms of $T_{j}^{i}$ as

$$
\frac{\mathrm{d}^{2} T_{j}^{i}}{\mathrm{~d} \tau^{2}}+R_{k l m}{ }^{i} v^{k} v^{m} T_{j}^{l}=-\Lambda^{i}{ }_{l} T_{j}^{l}=0 .
$$

Similarly, for the free particle one can obtain

$$
\begin{aligned}
\frac{\mathrm{d}^{2} \hat{T}_{j}{ }_{j}}{\mathrm{~d} \tau^{2}} & =-\hat{\Lambda}^{i}{ }_{l} \hat{T}^{l}{ }_{j}=0, \\
\hat{T}^{i}{ }_{j}\left(\tau_{1}\right) & =0, \quad \frac{\mathrm{d} \hat{T}^{i}{ }_{j}}{\mathrm{~d} \tau}\left(\tau_{1}\right)=\delta^{i}{ }_{j}
\end{aligned}
$$

from which the ratio in Eq. (30) can be rewritten as 15

$$
\left|\frac{\prod_{\alpha} \hat{\lambda}_{\alpha}}{\prod_{\alpha} \lambda_{\alpha}}\right|=\left|\frac{\operatorname{det} \hat{T}_{j}^{i}\left(\tau_{2}\right)}{\operatorname{det} T_{j}^{i}\left(\tau_{2}\right)}\right| \text {. }
$$

On the other hand, the partition function for a free particle is given as [16]

$$
\hat{Z}^{(2)}\left(q, \tau_{2} ; p, \tau_{1}\right)=(2 \pi i)^{-3 / 2}\left(\frac{\operatorname{det} \hat{g}_{i j}\left(\tau_{1}\right)}{\operatorname{det} \hat{T}_{j}^{i}\left(\tau_{2}\right)}\right)^{1 / 2} .
$$

Using the above results (39) and (40), the second order partition function (30) can be rewritten as

$$
Z^{(2)}\left(q, \tau_{2} ; p, \tau_{1}\right)=(2 \pi i)^{-3 / 2} e^{i \pi \sum_{\alpha}\left(\operatorname{sign} \lambda_{\alpha}-\operatorname{sign} \hat{\lambda}_{\alpha}\right) / 4}\left|\frac{\operatorname{det} \hat{g}_{i j}\left(\tau_{1}\right)}{\operatorname{det} T_{j}^{i}\left(\tau_{2}\right)}\right|^{1 / 2}
$$

to yield

$$
Z^{(2)}\left(q, \tau_{2} ; p, \tau_{1}\right)=(2 \pi i)^{-3 / 2} e^{i \pi \sum_{\alpha}\left(\operatorname{sign} \lambda_{\alpha}-\operatorname{sign} \hat{\lambda}_{\alpha}\right) / 4}\left|\operatorname{det} \hat{g}_{i j}\left(\tau_{1}\right) \frac{\partial v^{j}\left(\tau_{1}\right)}{\partial x^{k}\left(\tau_{2}\right)}\right|^{1 / 2} .
$$

\footnotetext{
${ }^{4}$ Since the coordinates $x^{i}(\tau)$ and the velocity $v^{i}(\tau)=\mathrm{d} x^{i} / \mathrm{d} \tau(\tau)$ are independent variables at the same time, say $\tau_{1}$, one can easily check that the definition (35) satisfies Eq. (34).
} 
Now we consider the Hamilton-Jacobi theory [17] where classical conjugate momentum $p_{i}^{c l}(\tau)$ corresponding to $x^{i}(\tau)$ in the action (11) is given as

$$
p_{i}^{c l}(\tau)=\frac{\partial L_{c l}}{\partial v^{i}}=g_{i j} v^{j}, \quad p_{i}^{c l}\left(\tau_{1}\right)=\frac{\partial S_{c l}}{\partial x^{i}\left(\tau_{1}\right)}
$$

from which we can obtain

$$
\begin{aligned}
\hat{g}_{i j}\left(\tau_{1}\right) & =\frac{\partial p_{i}^{c l}\left(\tau_{1}\right)}{\partial v^{j}\left(\tau_{1}\right)} \\
\frac{\partial v^{i}\left(\tau_{1}\right)}{\partial x^{j}\left(\tau_{2}\right)} & =\frac{1}{\hat{g}_{i k}\left(\tau_{1}\right)} \frac{\partial p_{k}^{c l}\left(\tau_{1}\right)}{\partial x^{j}\left(\tau_{2}\right)}=\frac{1}{\hat{g}_{i k}\left(\tau_{1}\right)} \frac{\partial^{2} S_{c l}}{\partial x^{k}\left(\tau_{1}\right) \partial x^{j}\left(\tau_{2}\right)}
\end{aligned}
$$

to, together with Eqs. (12) and (42), yield the desired semiclassical partition function

$$
Z\left(q, \tau_{2} ; p, \tau_{1}\right)=(2 \pi i)^{-3 / 2} e^{i \pi \sum_{\alpha}\left(\operatorname{sign} \lambda_{\alpha}-\operatorname{sign} \hat{\lambda}_{\alpha}\right) / 4} e^{i S_{c l}}\left|\operatorname{det} \frac{\partial^{2} S_{c l}}{\partial x^{i}\left(\tau_{1}\right) \partial x^{k}\left(\tau_{2}\right)}\right|^{1 / 2},
$$

where the determinant involved here is known as the Van Vleck determinant [18]. Here $\operatorname{sign} \hat{\lambda}_{\alpha}$ is easily checked to be positive and $\operatorname{sign} \lambda_{\alpha}$ could be positive or negative, so that the corresponding additional phase factor in the semiclassical partition function (45) is given by $e^{-i \mathcal{N} \pi / 2}$ where $\mathcal{N}$ is the number of negative eigenvalues, counted with their multiplicity [13, 14], of the operator $\Lambda$ in the eigenvalue equation (18) of the Morse theory.

In conclusion, we have explicitly derived the semiclassical partition function in the framework of the Morse theory, where we can introduce the Jacobi fields and the Hamilton-Jacobi theory to yield the phase factor of the semiclassical partition function associated with the eta invariant of Atiyah. It will be interesting, through further investigation, to study conjugate points [12] in the framework of this semiclassical approach to differential geometry.

The author would like to thank the hospitality of the C.N. Yang Institute for Theoretical Physics, SUNY at Stony Brook and the CIT-USC Center for Theoretical Physics, University of Southern California, where a part of this work has been done. He also would like to thank Itzhak Bars, John Milnor, Chiara Nappi, Peter van Nieuwenhuizen and Edward Witten for helpful discussions and concerns. This work is supported in part by Grant No. 2000-2-11100-002-5 from the Basic Research Program of the Korea Science and Engineering Foundation. 


\section{References}

[1] E. Witten, Comm. Math. Phys. 121 (1989) 351.

[2] S.T. Hong, Strangeness in Chiral Bag Model (Stony Brook, 1993) Ph.D Thesis.

[3] M. Anderson, Comparison Geometry (Cambridge Univ. Press, 1997) Vol. 30 pp. $49-82$.

[4] G.W. Gibbons, Class. Quantum Grav. 16 (1999) 1677; G.T. Horowitz, 1992 Trieste Spring School on String Theory and Quantum Gravity, hepth/9210119; G.J. Galloway, K Schleich, D. Witt and E. Woolgar, Phys. Rev. D60 (1999) 104039; M. Cai and G.J. Galloway, hep-th/0102149.

[5] R.P. Feynman, Rev. Mod. Phys. 20 (1948) 367.

[6] C. Morette, Phys. Rev. 81 (1951) 848.

[7] M.F. Atiyah, V. Patodi and I. Singer, Math. Proc. Camb. Phil. Soc. 77 (1975) 43; 78 (1975) 405; 79 (1976) 71.

[8] R.M. Wald, General Relativity (The Univ. of Chicago Press, 1984).

[9] R. Feynman and A.R. Hibbs, Quantum Mechanics and Path Integrals (McGraw-Hill, 1965).

[10] R.F. Dashen, B. Hasslacher and A. Neveu, Phys. Rev. D10 (1974) 4114, 4130, 4138; D11 (1975) 3424.

[11] R. Rajaraman, Solitons and Instantons (North-Holland, 1982).

[12] J. Milnor, Morse Theory (Princeton Univ. Press, 1963).

[13] M. Morse, The Calculus of Variables in the Large (Amer. Math. Soc., 1934).

[14] S. Levit and U. Smilansky, Ann. Phys. 103 (1977) 198.

[15] S. Levit and U. Smilansky, Proc. Amer. Math. Soc. 65 (1977) 299.

[16] G.J. Papadopoulos, Phys. Rev. D11 (1975) 2870. 
[17] H. Goldstein, Classical Mechanics (Addison-Wesley, 1980); I.S. Sokolnikoff, Tensor Analysis (John Wiley \& Sons, 1964).

[18] J.M. Van Vleck, Proc. Nat. Acad. Sci, 14 (1928) 178. 